\title{
PATTERN OF DISEASES AND OUTCOMES OF NEONATAL ADMISSION IN BENUE STATE UNIVERSITY TEACHING HOSPITAL, MAKURDI, NIGERIA
}

Ochoga MO ${ }^{I^{*}}$, Abah RO ${ }^{1}$, Idoko $A^{1}$, Dabit JO', Ebonyi $A^{2}$, Okpe ES ${ }^{2}$.

Department of Paediatrics, Benue State University Teaching Hospital, Makurdi ${ }^{1}$, and University of Jos/Jos University Teaching Hospital. ${ }^{2}$.

*Correspondence Author: Dr. Ochoga MO. Department of Paediatrics, Benue State University Teaching Hospital Makurdi. E-mail: omoochoga@yahoo.com

Received date: March $6^{\text {th }}, 2018$, Accepted date: April $9^{\text {th }}, 2018$. Published date: April $20^{\text {th }}, 2018$

\begin{abstract}
The burden of neonatal morbidity and mortality remains a major health challenge, and contributes to deaths among children under the age of five years especially in developing countries. The objective of this study was to report the pattern of diseases and outcomes of neonatal admission over a three-year period. This retrospective study was conducted in the Special Care Baby Unit from January 2013 to December 2015. Data of all the patients admitted to the unit during the study period was analyzed for sex, gestational age, weight, duration of stay, diagnosis on admission, age at admission, outcome and causes of death. A total of 505 patients were admitted during the study period, 310(61.4\%) were males and 195(38.6\%) were females. There were 374(74.1\%) term babies while 111(22.0\%) preterm and 20(4.0\%) were post term. Low birth weight accounted for 134(30.5\%) of all the patients. The commonest cause of neonatal admission was sepsis $40.8 \%$, followed by jaundice $21.0 \%$, and prematurity 14.1 $\%$. Of the total admissions $86.3 \%$ were successfully treated and discharged, $4.2 \%$ discharged against medical advice and $9.5 \%$ died. Neonatal deaths were commonly associated with birth asphyxia $31.3 \%$, followed by neonatal sepsis $27.1 \%$ and prematurity $25.0 \%$. Majority (44.6\%) of the infants were admitted on the second to seventh day of life. Improving antenatal services, delivery services and neonatal care will help to reduce neonatal morbidity and mortality.
\end{abstract}

Keywords: Birth Asphyxia, Neonates, Prematurity, Sepsis, SCBU.

\section{Introduction}

Worldwide, 5.9 million children die before reaching the age of 5 years and 2.7 millions of this die in the first 28 days of life. ${ }^{1}$ The global under-five mortality rate is currently $43 / 1000$ live births with a neonatal mortality rate of $19 / 1000$ live births in $2015 .{ }^{1}$ Substantial progress has been made in reducing child deaths since $1990 .{ }^{1}$ Both the number of under-five deaths and the rate of under-five mortality have fallen by more than half since $1990 .{ }^{1,2}$ Despite the global decline, neonatal deaths account for 44 percent of all under five deaths. ${ }^{2}$ Globally, major progress has been made in improving child survival but insufficient to meet the Millennium Development Goal 4(MDG4). ${ }^{2}$ The first 28 days of life, the neonatal period, is the most vulnerable time for a child's survival. ${ }^{3}$

Many deaths in the first month of life result from diseases and conditions that are preventable or treatable with proven, cost-effective interventions. Globally, preterm birth complications and complications during labour and delivery account for nearly 60 percent of neonatal deaths. ${ }^{2}$ One million babies die every year on their day of birth and more than a third of neonatal deaths take place on the day 
of birth. Close to 2 million babies die in the first week of life. ${ }^{2}$

The causes of neonatal deaths worldwide include prematurity (34\%), birth asphyxia (24\%), infections (22\%) and congenital malformations (9\%) all collectively account for $89 \%$ of neonatal deaths worldwide. ${ }^{1}$

Maternal and child health care services vary from place to place. Knowing the pattern of neonatal morbidity and mortality is a useful indicator of the availability, utilization, and effectiveness of such services. ${ }^{4}$ Information on admission and mortality of hospitalized neonates reflects the major causes of illnesses and standard of care provided to neonates in a particular locality. ${ }^{5}$ However, study on this is scanty in our environment. The purpose of this study was to review the pattern of diseases and outcomes of neonatal admissions and to influence positively neonatal health outcomes in our environment.

\section{Materials and Methods}

The study was undertaken at Benue State University Teaching Hospital (BSUTH), Makurdi, which became operational in the year 2012. Benue State has an estimated 2017 total population of 5,840,420 which is projected from 2006 population census and is a state in the middle-Belt region of Nigeria. It is a State owned tertiary health facility that serves all Benue State and other neighboring States of Kogi, Nasarawa, Ebonyi and Cross River.

The SCBU is divided into an out born unit comprising of 4 cots, 2 incubators, 1 resuscitaire and two phototherapy units and the inborn unit which is equipped with 4 cots, 2 incubators, 4 phototherapy units, 2 oxygen cylinders, 1 resuscitaire and 1 apnoea monitor. The unit is manned by 1 Paediatrician, Paediatric residents and Nursing staff.

The study was a retrospective descriptive study based on analysis of the record of all patients admitted into the special care baby unit (SCBU) of BSUTH, from January 2013 to December 2015.The data of all the admitted neonates were analyzed and patients whose information was incomplete were excluded from the study. A total of 505 eligible cases of admission in the SCBU during the study period were reviewed. The information collected included age of the neonate on admission, sex, gestational age at birth, weight on admission or at birth, duration of hospital stay, final diagnosis and outcomes (discharge, discharged against medical advice, death) were analyzed using Statistical Package for Social Sciences (SPSS) IBM version 23.0 was used for analysis.

The diagnosis of prematurity and low birth weight was based on the World Health Organization definitions. Prematurity is described as live newborn delivered before 37 weeks from $1^{\text {st }}$ day of last menstrual period, low birth weight (LBW) as having a birth weight less than $2500 \mathrm{~g}$, very low birth weight(VLBW) is a birth weight of $<1500 \mathrm{~g}$ and extremely low birth weight (ELBW) as a birth weight of $<1000 \mathrm{~g}$. Sepsis was suspected on clinical grounds and confirmed by relevant investigations. Birth asphyxia and congenital anomalies were diagnosed on clinical grounds. Neonatal jaundice was diagnosed on clinical grounds and confirmed with relevant clinical investigations. Approval for the study was obtained from the BSUTH Health Research Ethical Committee.

\section{Results}

Of the 505 cases reviewed, 310 were male $(61.4 \%)$ while $195(38.6 \%)$ were female. Majority (78.9\%) of the neonates were admitted during the first week of life. Regarding the birth weight of these babies, 374 patients were categorized as having normal birth weight $(62.6 \%), 154(30.5 \%)$ as LBW and $35(6.9 \%)$ as large for gestational age (LGA) (6.9\%). Other variables related to admission are shown in Table 1. Major causes of admission were sepsis (40.8\%), neonatal jaundice $(21.0 \%)$, prematurity $(14.1 \%)$. Other causes include congenital anomaly (11.5\%) and birth asphyxia (10.9\%). [Table.2]. Neonates admitted age less than 24 hours of age were $34.3 \%$ while age at 2-7 days $44.6 \%$ and 8-28 days constitutes $21.2 \%$. (Figure 1)

Of the 505 admissions, 436(86.3\%) were discharged in a satisfactory condition, 21(4.2\%) were discharged against medical advice and 48(9.5\%) died (Figure 2). The commonest cause of mortality was birth asphyxia in $(15,31.3 \%)$, followed by NNS (13, $27.1 \%)$, prematurity $(12,25.0 \%)$, congenital anomaly $(7,14.6 \%)$ and jaundice $2.1 \%$. (Table.3). 
Table 1: Demographic Characteristics of Neonates

\begin{tabular}{|c|c|c|}
\hline Variables & Number (N) & $\%$ \\
\hline \multicolumn{3}{|l|}{$\overline{\text { Sex }}$} \\
\hline Male & 310 & 61.4 \\
\hline Female & 195 & 38.6 \\
\hline \multicolumn{3}{|l|}{ Gestational Age at birth } \\
\hline Term & 374 & 74.1 \\
\hline Preterm & 111 & 22.0 \\
\hline Post term & 20 & 4.0 \\
\hline Mean Age & $36.6( \pm 1.6)$ years & \\
\hline \multicolumn{3}{|l|}{ Birth weight } \\
\hline $\operatorname{Normal}(2.5-4.0 \mathrm{Kg})$ & 316 & 62.6 \\
\hline $\mathrm{LBW}(<2.5 \mathrm{Kg})$ & 154 & 30.5 \\
\hline $\mathrm{LGA}(>4 \mathrm{Kg})$ & 35 & 6.9 \\
\hline Mean Birth weight & $3.1( \pm 1.6) \mathrm{Kg}$ & \\
\hline \multicolumn{3}{|l|}{ Duration of Stay (Days) } \\
\hline$\leq 7$ & 242 & 47.9 \\
\hline $8-14$ & 172 & 34.1 \\
\hline $15-28$ & 66 & 13.1 \\
\hline$\geq 29$ & 25 & 5.0 \\
\hline Mean Duration of Stay & $8( \pm 1.8)$ days & \\
\hline
\end{tabular}

Table 2: Causes of Neonatal Admissions

\begin{tabular}{lcc}
\hline Diagnosis & Number $\mathbf{( N )}$ & \% \\
\hline Sepsis & 206 & 40.8 \\
Jaundice & 106 & 21.0 \\
Prematurity & 71 & 14.1 \\
Congenital Anomaly/Defect & 58 & 11.5 \\
Birth Asphyxia & 55 & 10.9 \\
Meconium Aspiration Syndrome & 7 & 1.4 \\
Neonatal Tetanus & 2 & 0.4 \\
\hline
\end{tabular}

Table 3: Causes of Neonatal Deaths

\begin{tabular}{lcc}
\hline Diagnosis & Death(N) & $\%$ \\
\hline Birth Asphyxia & 15 & 31.3 \\
Sepsis & 13 & 27.1 \\
Prematurity & 12 & 25.0 \\
Congenital Anomaly/Defect & 7 & 14.6 \\
Jaundice & 1 & 2.1 \\
\hline
\end{tabular}




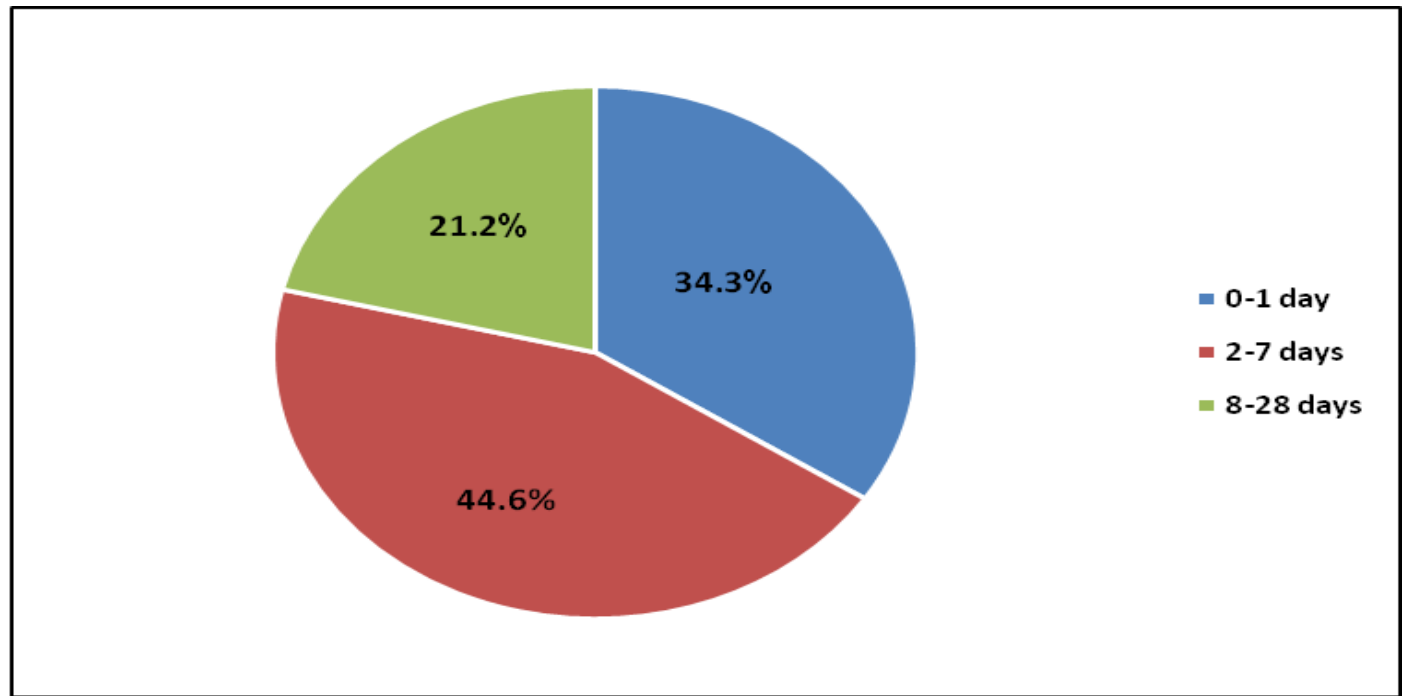

Figure1: Age at Admission of Neonates

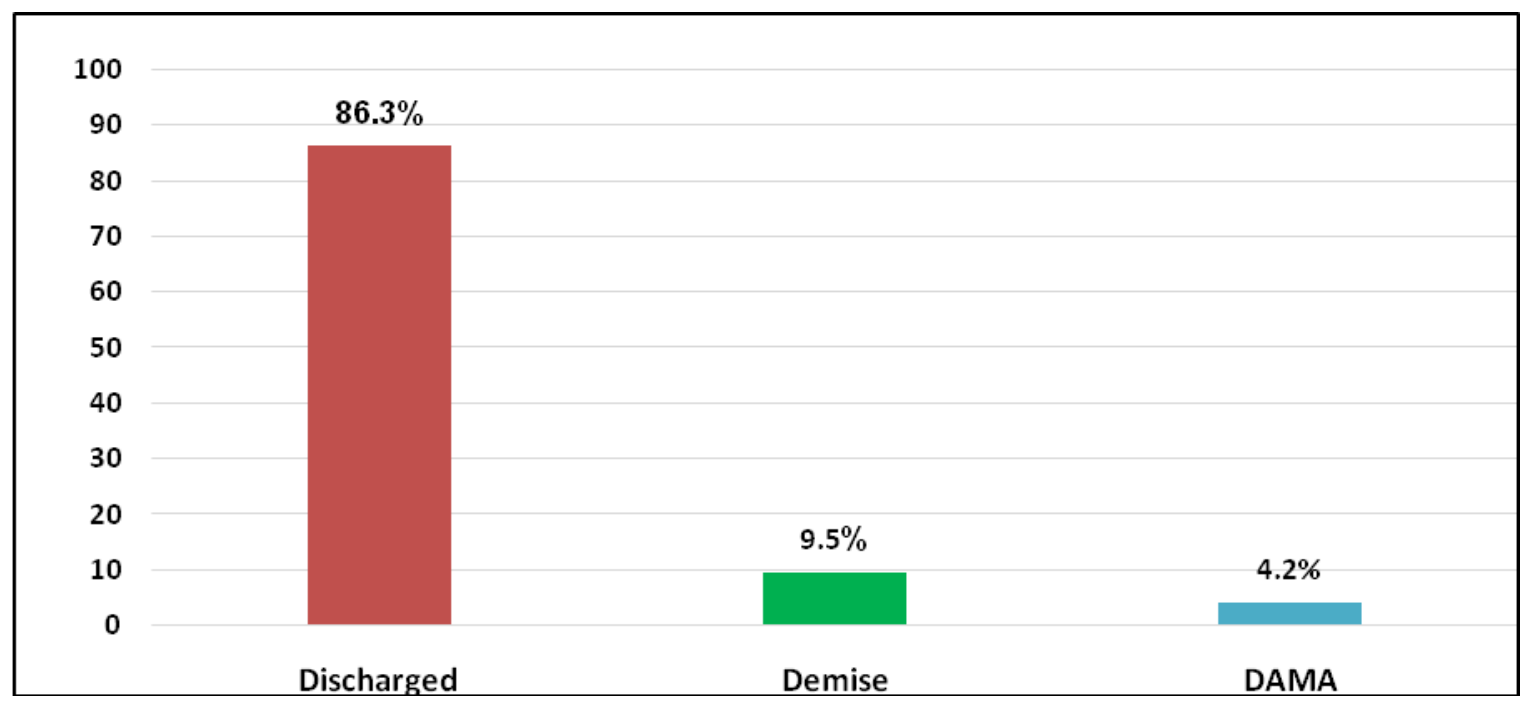

Figure 2: Treatment Outcome of Neonatal Admissions 


\section{Discussion}

This study assessed the pattern of diseases and outcomes of neonatal admissions. The number of neonatal admissions in our study is low $^{6}$ as most of the deliveries are being conducted at home, with traditional birth attendants and the fact that our facility is still very young. The study revealed that more males $(61.4 \%)$ were admitted when compared to females $(38.6 \%)$.This may be related to the biological vulnerability of males to infections. A recent study has established the genetic influence in neonatal infection, revealing that $\mathrm{x}$ chromosomelinked diseases are predominant in males than females. $^{7}$

This is comparable to other studies where 1.25:1 in favour of males in Kano, ${ }^{8}$ males $53.7 \%$ versus 46.3 $\%$ females in Abuja, ${ }^{9} 54.6 \%$ males vs $45.4 \%$ females in Benin, ${ }^{10} 57.7 \%$ males vs $42.3 \%$ females in Jos, ${ }^{11}$ and $63.0 \%$ males vs $37.0 \%$ females in Pakistan. ${ }^{12}$ In our study, LBW was found in $30.5 \%$ of patients; this is at variance to $22.4 \%$ documented by Toma and colleagues, ${ }^{11} 20.5 \%$ by Hamid et al ${ }^{14}$ in Bangladesh and $24.5 \%$ by Ekwochi et al in 2014. ${ }^{15}$ However, higher prevalence of LBW were reported byMuktarYola et al in Kano $(32.1 \%),{ }^{8}$ Oke Chukwu and colleagues in Abuja (32.7\%), ${ }^{9}$ Omoigberale in Benin reported $(26.1 \%),{ }^{10}$ and Ali et al in Pakistan reported a prevalence of $37.7 \% .^{12}$

This can be explained by the institutions dealing with a much larger number of high risk pregnancies leading to higher numbers of LBW babies as compared to our hospital. Our findings are similar with what has been reported for more than 25 years. ${ }^{13}$ By implication further strategies of management of pregnancies and deliveries should be looked into in order to reduce the incidence of babies been born low birth weight in our environment. This can be achieved by strengthening capacity building of health providers at all levels of health care.

In this study, sepsis 206 (36.0\%) was the commonest cause of admission, resulting in $27.1 \%$ of neonatal deaths. Toma et al in 2013 showed that $37.1 \%$ of neonatal admissions in Jos was due to sepsis, ${ }^{11}$ and Omoigberale in Benin reported sepsis as one of the most common morbidity suffered by the neonates. ${ }^{10}$ A similar study done in Jos in 2014 reported a prevalence of neonatal infections to be $34.4 \%{ }^{16}$
Majority of deliveries in Nigeria still take place outside the healthcare facilities, with poor environmental conditions. ${ }^{6}$ This may have contributed to the high prevalence of sepsis in this study ${ }^{3}$ Neonatal sepsis continues to be a major cause of morbidity and mortality in Nigeria. It is also one of the major causes of neonatal mortality in developing countries in general. Similar studies in different parts of Nigeria reported birth asphyxia as the commonest cause of neonatal admission, ${ }^{8,15}$ unlike in the present study where it accounted for $10.9 \%$ of admissions. The contribution of birth asphyxia to total admission was less in this study possibly because asphyxiated babies die at home or do not make it to the hospital considering the bad roads and long distance of travel.

Neonatal jaundice was the second leading cause of admissions compared to the study by Sain $\mathrm{N}$ et al in India which reported neonatal jaundice (NNJ) (7.0\%) as the commonest cause of neonatal admissions. ${ }^{17}$ Information about the incidence of $\mathrm{NNJ}$ in developing countries is lacking as the vast majority of births occur at home. In this study prematurity was the reason for admission in $22.0 \%$ of neonates and is consistent with the high rates of $20.1 \%$ and lower than $40.0 \%$ reported by Toma et $\mathrm{al}^{11}$ and Ibe et al. ${ }^{18}$, respectively. The low figure in this study could be due to the fact that our hospital is not the primary referral center for newborns from rural areas.

Congenital anomalies in this study contributed to $11.5 \%$ of admissions. Other authors from different centers had documented various congenital anomalies which were not detected during the antenatal period. $^{19,20,21,22}$

Our study showed that $44.6 \%$ patients were admitted between the second and seventh day of life. This could be explained by the fact that most of the deliveries in Benue State and other Northern States of Nigeria occurred outside health facilities. ${ }^{6}$ The outcome in our study was good as $86.3 \%$ of patients were discharged. Globally the commonest causes of neonatal mortality are prematurity with its complications, birth asphyxia, sepsis and severe congenital malformations. ${ }^{1}$ The commonest cause of mortality in our study was birth asphyxia followed by sepsis, prematurity, congenital anomalies, and neonatal jaundice. Birth asphyxia was also reported to be the commonest cause of death in other studies. ${ }^{9,15,16}$ 
The high neonatal mortality due to birth asphyxia could be as a result of poor antenatal services and late referral of both high-risk pregnancies and sick newborns.

A major limitation of the study was the fact that the authors were not in control over the outcome. About thirty patients had incomplete data and were excluded thereby reducing the size and power of the study.

A protocol is being developed for the SCBU and this will improve the quality of care for the patients. We need to get the appropriate technology tools to assist in data capturing.

\section{Conclusion}

In our study, sepsis, jaundice, prematurity, congenital anomalies and birth asphyxia were the main causes of admission.

These conditions birth asphyxia, prematurity, and sepsis were associated with a majority of the case fatalities. Most of the causes of morbidity and mortality are preventable. The number of admissions due to these causes could be reduced by encouraging proper antenatal care, delivery services and post natal care of all pregnancies to health facilities. It is recommended that proper and timely referral of all high risk pregnancies to tertiary centers be advocated.

\section{References}

1. United Nations Children's Fund. Committing to Child survival: A Promise Renewed. New York: United Nations Children's Fund (UNICEF);2015.

2. United Nations. The millennium development goals report. New York. United Nations; 2014.

3. Lawn JE, Cousens S, ZupanJ.Four million neonatal deaths: When? Where? Why? Lancet. 2005;365:891-900.

4. The Nigerian Academy of Science. Reducing Maternal and Infant Mortality in Nigeria (Workshop Summary). Imo: NwosuJ, OdubanjoMO, OsinusiBO, editors.West African Book Publishers, Lagos, Nigeria, 2009.

5. Hoque M, Haaq S, Islam R. Causes of neonatal admissions in KwaZulu-Natal, South Afr J Epidemiol Infect 2011: 26:26-9.
6. Federal Ministry of Health. National Demographic and Health Survey. Abuja: FMOH;2012.

7. Libert C, Dejager L, Pinheiro I (2010) The X chromosome in immune functions: when a chromosome makes the difference. Nat Rev Immunol 10:594-604.

8. Mukhtar-Yola M, Iliyasu Z. A review of neonatal morbidity and mortality in Aminu Kano Teaching Hospital, Northen Nigeria. Trop Doct. 2007; 37:130-2.[PubMed].

9. Okechukwu AA, Achonwa A. Morbidity and mortality patterns of admissions into the Special Care Baby Unit of University of Abuja Teaching Hospital, Gwagwalada, Nigeria. Niger J ClinPract 2009; 12:389-94.[PubMed].

10. Omoigberale AI, Sadoh WE, Nwaneri DU. A 4 year review of neonatal outcome at the Univeristy of Benin Teaching Hospital, Benin City. Niger J ClinPract 2010; 13:321-5.

11. Toma BO, Ige OO, Abok II, Onmuanaku C, Abah RO, Donli A. Pattern of neonatal admissions and outcome in a tertiary institution in North Central Nigeria. Journal of Medicine in the Tropics 2013; 15:121-125.

12. Ali SR, Ahmed S, Lohana H. Disease Patterns and Outcomes of Neonatal Admissions at a Secondary Care Hospital in Pakistan.SultanQaboosUniv Med J. 2013;13:424-428.

13. Lawn JE, Cousens SN, Darmstadt GL, Bhutta ZA, Martines J, Paul V,etal.One year after The Lancet Neonatal Survival Series. Was the call for action heard? Lancet 2006, 367:541-7. [PubMed].

14. Hamid F, Quaium MA, Rahman A, Ahmad AR, Khan S, Hussain T, et al. Audit of Neonatal Morbidity and Mortality in A Tertiary Care Hospital,Sylhet. J of ChattagramMaa-O-Shishu Hospital Medical College 2016; 15:45-48.

15. Ekwochi U, NduIK,Nwokoye IC, Ezenwosu OU, Amadi OF, Osuorah D. Pattern of morbidity and mortality of newborns admitted into the Sick and Special Care baby unit of Enugu State University Teaching Hospital,EnuguState.Niger J ClinPract 2014; 17:346-51.

16. Bode -Thomas F, IkehEI, Pam SD, Ejeliogu EU. Current aetiology of neonatal sepsis in Jos 
University Teaching Hospital. Niger J Med 2014; 13:130-5.

17. Sain N, Chhabra S, Chhabra S, Garg L, Garg N. Pattern of neonatal morbidity and mortality: A prospective study in a District Hospital in Urban India. J Clin Neonatal 2016; 5:183-8.

18. Ibe BC, Ibeziako SN, Azubuike JC. A study of neonatal admissions into a newborn special unit. Niger J Paediatr 1994; 21:20-5.

19. Abdellatif M, Ahmed M, BataclanMF,Khan AA Al BattashiA,AlManin A. The Patterns and Causes of Neonatal Mortality at a Tertiary Hospital in Oman. Oman Med J 2013; 28:422426.

20. Pam S, Bode -Thomas F, Isaac WE, Ibanga HB, Adekwu OA, TomaBO, et al. Are congenital anomalies common in Jos Nigeria? Highland Med Res J 2004; 2:19-28.

21. Chirdan LB, Uba AF, Pam SD, Edino ST, Mandong BM, Chirdan OO.

Sacrococcygealteratoma.Clinical characteristics and long-term outcome in Nigerian children. Ann Afr Med 2009; 8:105-9. [PubMed].

22. Chirdan LB, Uba F, Pam S. Intestinal Atresia: Management problems in a developing Country. PaediatrSurgInt 2004; 20:834-7. 